\title{
Re: Perioperative Complications after Living Kidney Donation: A National Study
}

\author{
Lentine KL1, Lam NN2, Axelrod D3, Schnitzler MA1, Garg AX4, Xiao H1, Dzebisashvili N3, Schold JD5, \\ Brennan DC6, Randall H1, King EA7, Segev DL7
}

1Saint Louis University Faculty of Medicine, Center for Abdominal Transplantation, Missouri, USA

2University of Alberta, Edmonton Department of Nephrology, Canada, North America

3Dartmouth Hitchcock Medical Center, Department of Surgery, Clinic of Abdominal Transplantation, Dartmouth, USA

4 Western University Faculty of Medicine, Department of Nephrology, Canada, North America

${ }^{5}$ Cleveland Clinic Department of Quantitative, Health Sciences, Ohio, USA

6Washington University Faculty of Medicine, Department of Transplant Nephrology, Missouri, USA

7 Johns Hopkins Faculty of Medicine, Department of Surgery, Clinic of Transplantation, Maryland, USA

Am J Transplant 2016;16:1848-1857. doi: 10.1111/ajt.13687. Epub 2016 Mar 10.

\section{EDITORIAL COMMENT}

The authors have investigated the perioperative complications after donor nephrectomy integrating the US transplant registry with administrative records from an academic hospital consortium (97 centers, 2008-2012). 14.964 patients were verified as live donors through linkage with the Organ Procurement and Transplantation Network registry. Overall, 16.8\% of donors experienced a perioperative complication, including Clavien grade 2 or higher events in 8.8\%, Clavien grade 3 or higher in $7.3 \%$, and Clavien grade 4 or higher events in 2.5\%. The most common complications were gastrointestinal (4.4\%), bleeding (3.0\%), respiratory (2.5\%), and surgical/anesthesia-related injuries (2.4\%). After adjustment for demographic and clinical factors, African American donors were $26 \%$ more likely to experience any perioperative complication and 56\% more likely to experience the most severe complications. Other factors associated with increased risk of any perioperative complication, and with the most severe complications included predonation hematologic and psychiatric conditions and more recent years of donation. Donation at centers with the highest annual volume of living donor nephrectomies (>50 cases/year) was associated with approximately $45 \%$ lower risk of any perioperative complication and of the most severe complications. Donors who underwent robotic nephrectomy were twice as likely to experience severe perioperative complications (adjusted odds ratio 2.07 for Clavien grade 4 or higher events). To conclude, the authors found that while one in six US living kidney donors experienced a perioperative complication, the most severe complications were infrequent, affecting only $2.5 \%$ of donors.

Serkan Akıncı, MD

\section{Re: Timing of Pregnancy after Kidney Transplantation and Risk of Allograft Failure}

\author{
Rose C1, Gill J1,2, Zalunardo N1, Johnston O1, Mehrotra A3, Gill JS1,2,4 \\ 1 University of British Columbia, Department of Nephrology, Canada, North America \\ 2 University of British Columbia, Centre for Health Evaluation and Outcomes Sciences, Canada, North America \\ 3 Mount Sinai Faculty of Medicine, Department of Nephrology, New York, USA \\ 4Tufts-New England Medical Center, Massachusetts, USA
}

Am J Transplant 2016;16:2360-2367. doi: 10.1111/ajt.13773. Epub 2016 Apr 4.

\section{EDITORIAL COMMENT}

The authors have investigated the risk of allograft failure due to the timing of pregnancy after kidney transplantation. Of the 21.814 women aged 15-45 who have received a first kidney-only transplant in the United States Renal Data System, 729 pregnancies were identified using medicare claims. In multivariate analyses, pregnancy in the first posttransplant year was associated with an increased risk of allograft failure from any cause including death (ACGL) (HR: 1.18) and death censored graft loss (DCGL) (HR: 1.25), while pregnancy in the second posttransplant year was associated with an increased risk of DCGL (HR: 1.26). Pregnancy in the third posttransplant year was not associated with an increased risk of ACGL or DCGL. The cause of allograft failure was limited by incomplete data, but changes in immunosuppressant medications and unstable drug levels leading to acute and chronic rejection may be in the causal pathway resulting in graft loss after pregnancy. These results may be useful for physicians providing counseling to women wanting to conceive after transplantation. 